\title{
Atividades biológicas de Agave sisalana com ênfase para a ação antimicrobiana: uma
}

\section{revisão da literatura}

\author{
Biological activities of Agave sisalana with an emphasis on antimicrobial action: a literature review \\ Actividades biológicas de Agave sisalana con énfasis en la acción antimicrobiana: una revisión de la
}

literatura

Recebido: 06/02/2021 | Revisado: 11/02/2021 | Aceito: 24/02/2021 | Publicado: 03/03/2021

Jocinede da Silva Carneiro

ORCID: https://orcid.org/0000-0002-8959-3302 Unidade de Ensino Superior de Feira de Santana, Brasil E-mail: jocy_sc@hotmail.com

Maria Cristina de Jesus Silva

ORCID: https://orcid.org/0000-0003-4661-4182 Unidade de Ensino Superior de Feira de Santana, Brasil E-mail: c_tinacris@hotmail.com

Ediane Nascimento dos Santos

ORCID: https://orcid.org/0000-0002-3404-5587 Unidade de Ensino Superior de Feira de Santana, Brasil

E-mail: ediane.lufarma@gmail.com

Felicson Leonardo Oliveira Lima

ORCID: https://orcid.org/0000-0001-5256-6768

Faculdade Nobre de Feira de Santana, Brasil

E-mail: felicsonleonardo@hotmail.com

Misael Silva Ferreira Costa

ORCID: https://orcid.org/0000-0001-8042-2489

Faculdade Nobre de Feira de Santana, Brasil

Universidade Federal de Pernambuco, Brasil

Universidade Estadual de Feira de Santana, Brasil E-mail: ffcost@hotmail.com

\begin{abstract}
Resumo
Objetivo: Relatar as principais atividades biológicas de Agave sisalana, descrevendo detalhes de sua classificação botânica, aspectos da composição química e bioativa, além de destacar seu uso como antimicrobiano. Metodologia: Trata-se de uma revisão da literatura de caráter descritiva com abordagem qualitativa, elaborada por meio da análise de materiais indexados nas plataformas: SciELO, PubMed e o portal de periódicos da CAPES, publicados entre os anos de 2002 a 2021, redigidos nos idiomas: português, inglês e espanhol. Após aplicação dos critérios de inclusão e exclusão, bem como leitura dos materiais, 38 conteúdos foram selecionados para a composição do presente estudo. Resultados e discussão: A atividade bioativa de Agave sisalana, está vinculada aos componentes do seu metabolismo secundário, onde podem ser mensurados: derivados alcaloides, cumarinas, taninos e saponínicos. No que se refere as saponinas do sisal, estas são apontadas como detentoras de ação antifúngica e antibacteriana, principalmente contra microrganismos patogênicos. Além das atribuições mencionadas, os estudos vêm mostrando seus efeitos como: inseticida, larvicida, ovicida, antiparasitário, antioxidante, imunomodulador, analgésico, anti-inflamatório, sob a atividade comportamental e neuroquímica, bem como na quimioprevenção e controle do câncer. Conclusão: Variadas são as aplicações da Agave sisalana, seja ao âmbito industrial, artesanal terapêutico ou na agropecuária. Sua atividade antimicrobiana é cientificamente comprovada, seja na inferência de fungos ou bactérias, fazendo desta uma importante alternativa, frente a incidência de microrganismos resistentes.
\end{abstract}

Palavras-chave: Agave sisalana; Saponinas; Ação antimicrobiana.

\begin{abstract}
Objective: To report the main biological activities of Agave sisalana, describing details of its botanical classification, aspects of chemical and bioactive composition, in addition to highlighting its use as an antimicrobial. Methodology: This is a literature review of a descriptive character with a qualitative approach, elaborated through the analysis of materials indexed in the platforms: SciELO, PubMed and the portal of CAPES journals, published between the years 2002 to 2021, written in languages: Portuguese, English and Spanish. After applying the inclusion and exclusion criteria, as well as reading the materials, 38 contents were selected for the composition of this study. Results and discussion: The bioactive activity of Agave sisalana, is linked to the components of its secondary metabolism, where it can be measured: alkaloids, coumarins, tannins and saponins. With regard to sisal saponins, they are identified as
\end{abstract}


having antifungal and antibacterial action, especially against pathogenic microorganisms. In addition to the actions mentioned, studies have shown their effects such as: insecticide, larvicide, ovicide, antiparasitic, antioxidant, immunomodulator, analgesic, anti-inflammatory, under behavioral and neurochemical activity, as well as in chemoprevention and cancer control. Conclusion: The applications of Agave sisalana are varied, whether in the industrial, therapeutic artisanal or in agriculture. Its antimicrobial activity is scientifically proven, whether in the inference of fungi or bacteria, making this an important alternative, in view of the incidence of resistant microorganisms.

Keywords: Agave sisalana; Saponins; Antimicrobial Action.

\section{Resumen}

Objetivo: Informar las principales actividades biológicas de Agave sisalana, describiendo detalles de su clasificación botánica, aspectos de composición química y bioactiva, además de destacar su uso como antimicrobiano. Metodología: Se trata de una revisión bibliográfica de carácter descriptivo con enfoque cualitativo, elaborada a través del análisis de materiales indexados en las plataformas: SciELO, PubMed y el portal de revistas CAPES, publicada entre los años 2002 a 2021, redactada en idiomas: portugués, Inglés y español. Tras aplicar los criterios de inclusión y exclusión, además de la lectura de los materiales, se seleccionaron 38 contenidos para la composición de este estudio. Resultados y discusión: La actividad bioactiva de Agave sisalana, está ligada a los componentes de su metabolismo secundario, donde se puede medir: alcaloides, cumarinas, taninos y saponinas. Con respecto a las saponinas de sisal, se identifican por tener acción antifúngica y antibacteriana, especialmente contra microorganismos patógenos. Además de las acciones mencionadas, estudios han demostrado sus efectos como: insecticida, larvicida, ovicida, antiparasitario, antioxidante, inmunomodulador, analgésico, antiinflamatorio, bajo actividad conductual y neuroquímica, así como en quimioprevención y control del cáncer. Conclusión: Las aplicaciones de Agave sisalana son variadas, ya sea en el ámbito industrial, terapéutico artesanal o en la agricultura. Su actividad antimicrobiana está científicamente probada, ya sea en la inferencia de hongos o bacterias, lo que la convierte en una alternativa importante, dada la incidencia de microorganismos resistentes.

Palabras clave: Agave sisalana; Saponinas; Acción Antimicrobiana.

\section{Introdução}

Agave sisalana perrine, é uma planta de origem mexicana, pertencente à família Agavaceae. Historicamente, em meados de 1903 as primeiras mudas dessa planta chegaram ao Brasil, especificamente na Bahia. Trata-se da principal fibra dura produzida em todo o mundo, de cultivo essencial para pequenos produtores das vertentes do trabalho familiar. Além de qualificar-se como fonte de emprego e renda para muitos trabalhadores, a sua cultivação auxilia na fixação do homem na região semiárida do Nordeste, uma vez que esta, na maioria das vezes, é a única forma de plantio com retorno econômico satisfatório (Martins et al., 2009; Carneiro, 2016).

O gênero Agave circunda diversas espécies de interesse cultural, econômico e medicinal. No Brasil, a espécie com maior aproveitamento é a Agave sisalana, visto que dela extrai-se o Sisal. Espécie tipicamente tropical de melhor desenvolvimento nas regiões semiáridas, demonstrando resistência à seca. Os principais estados produtores de sisal são a Paraíba e a Bahia, sendo este último, detentor da região sisaleira, que engloba cidades como Retirolândia, Conceição do Coité, Valente, São Domingos, Santa Luz e Queimadas (Botura et al., 2013; Vuorinne et al., 2021).

Das folhas da A. sisalana são extraídas fibras, que compõe cerca de 3 a $5 \%$ da planta, em contrapartida, 95 a $97 \%$ restante, caracterizam-se como resíduos do beneficiamento, que por muito tempo foram descartados pelos produtores, porém, nos últimos anos vem sendo objeto de estudo pela sua vasta possibilidade em uso, seja em rações de animais, como adubo orgânico, ou pela indústria farmacêutica. Dentre as aplicações industriais das fibras do sisal, pode-se citar: uso na indústria automobilística, no artesanato, na fabricação de barbantes, cordas, cabos marítimos, sacos, tapetes, estofados, vassouras e pasta celulósica (Debnath et al., 2010; Cardoso, 2019).

A composição química da fibra de A. sisalana apresenta: celulose, hemicelulose, lignina e pectina (Martin et al., 2009). A composição bioativa, por sua vez, abriga compostos fenólicos, terpenos e saponinas (Santos et al., 2015).

Além de possuir diversas utilidades industriais, a conformação química da Agave sisalana confere-lhe multiplicidade terapêutica. No que se remete a sua atividade antimicrobiana esta é capaz de inferir a integridade membranar de fungos e 
bactérias (Gram positivas ou Gram negativas), promovendo a sua inativação e consequente morte (López-Romero et al., 2018).

A utilização terapêutica de produtos que provem da natureza, faz-se tão antiga quanto a civilização humana, onde em tempos remotos, produtos de origem mineral, vegetal e animal, foram úteis para a promoção da saúde. As plantas medicinais no decorrer da história destacaram-se como as principais mantenedoras da fitoterapia, sendo consideradas pela Organização Mundial da Saúde (OMS), como instrumento de importância para a assistência farmacêutica (Abreu, 2013).

Mediante afirmativas, o presente estudo objetivou relatar as principais atividades biológicas de Agave sisalana, descrevendo detalhes de sua classificação botânica, aspectos da composição química e bioativa, além de destacar seu uso como antimicrobiano.

\section{Metodologia}

A definição de pesquisa bibliográfica ou estudo de revisão descrita por Pereira (2018), é apontada como uma investigação executada por meio do uso de fontes secundárias de informações. Para que haja uma construção de excelência, requer-se a análise minuciosa de pesquisas relatadas por meio de estudos anteriores. Dentre as possíveis fontes de pesquisa pode-se utilizar de: artigos, dissertações, teses e livros, bem como outros materiais de respaldo científico.

O presente estudo enquadra-se nos dogmas metodológicos de uma revisão da literatura de caráter descritiva com abordagem qualitativa, elaborada por meio da averiguação crítica de materiais indexados nas plataformas: SciELO, PubMed e o portal de periódicos da CAPES. A busca foi realizada sob uso dos termos: Agave sisalana; Saponinas; Ação antimicrobiana, em um recorte temporal de 19 anos (2002-2021), pela insuficiência de conteúdos recentes que atendessem aos objetivos do estudo.

Para a execução da pesquisa, critérios de inclusão e exclusão foram aplicados. Dentre os critérios de inclusão, foram abrigados materiais com teor de originalidade, abordando o tema proposto, atendendo aos objetivos e recorte temporal do estudo, além de sua descrição pertencer aos idiomas: português, inglês ou espanhol. No que se destina aos critérios de exclusão, estes foram aplicados em materiais duplicados, com idiomas diferentes dos pré-estabelecidos e fora do recorte temporal.

A busca foi realizada inicialmente pela leitura exploratória (I); posteriormente foi feita a leitura seletiva (II); e por fim, a seleção dos itens aprovados (III). Em suma, foram triados para a leitura inicial 106 artigos, destes, 52 foram encaminhados para a leitura seletiva e apenas 38 foram então selecionados mediante leitura na íntegra, para a composição do presente estudo.

\section{Resultados e Discussão}

\subsection{Classificação botânica da Agave sisalana}

Souza-Júnior (2017) explana que o gênero Agave engloba cerca de 300 espécies, estas nativas da América do Norte e do Sul. Contudo, apenas duas espécies ganham ênfase no âmbito comercial de produção das fibras, sendo: A. fourcroydes e A. sisalana. O sisal é uma planta detentora de folhas lisas, rígidas e com coloração verde brilhante, possui um formato comparado a uma espada com tamanho varável em largura (10 a $15 \mathrm{~cm}$ ) e comprimento (1 a 2 metros), a extremidade da folha abriga um espinho de cor marrom e arredondado, com 20 a $25 \mathrm{~cm}$ de cumprimento. Cada planta consegue produzir por ano, entre 10 a 40 folhas, estas por sua vez demonstram esteticamente uma inclinação múltipla em relação ao eixo vertical da planta. A mesma é acaulescente, apresentando apenas um tronco principal que pode chegar a 1,2 metros de comprimento e $20 \mathrm{~cm}$ de diâmetro. Na Tabela 1 há a descrição taxonômica da planta de estudo. 
Tabela 1: Classificação toxonômica da Agave sisalana.

\begin{tabular}{cc}
\hline Domínio & Eukaryota \\
\hline Reino & Plantae \\
\hline Filo & Spermatophyta \\
Subfilo & Angiospermae \\
\hline Classe & Monocotiledonae \\
\hline Ordem & Liliales \\
\hline Família & Agavaceae \\
\hline Subfamília & Agavoideae \\
\hline Gênero & Agave \\
\hline Subgênero & Euagave \\
\hline Espécie & Sisalana
\end{tabular}

Fonte: Adaptado de Barreto (2017).

\subsection{Composição química da Agave sisalana}

Martin e colaboradores (2009), caracterizam a composição química da fibra do sisal, como possuinte de 65,8\% de celulose, $12 \%$ de hemicelulose, 9,9\% de lignina e 0,8\% de pectina. Entretanto, Mwaikambo et al., (2002) apresenta outros percentuais, como $73 \%$ de celulose, $13 \%$ de hemicelulose, $11 \%$ de lignina e $2 \%$ de pectina.

Barreto (2017) qualifica o resíduo das folhas de Agave sisalana quanto a sua composição, detalhando a presença de: água, celulose, parênquima, compostos inorgânicos, componentes do metabolismo primário e secundário da planta, além de fibras.

Os autores Branco et al., (2010), Lin (2014) e Maran \& Priya (2015) por sua vez, complementam que além destes componentes, há a presença de D-manitol e uma variedade de carboidratos, como pectinas e polissacarídeos, constituídos dos ácidos galacturônicos, arabinose, galactose e ramnose. Santos et al., (2015) especifica a presença de saponinas, compostos fenólicos e terpenos na A. sisalana.

\subsection{Composição bioativa e atividades biológicas de Agave sisalana}

A eficácia de um tratamento mediado por plantas medicinais, seja em animais ou em humanos, se dá, pelas propriedades que essas plantas produzem a partir do seu próprio metabolismo, o que resulta em substancias com capacidade de modular funções fisiológicas e orgânicas de outros seres vivos (Almeida, 2013).

Taiz \& Zeiger (2013) descrevem que uma planta pode produzir dois tipos de metabólitos: primários e secundários. No que se refere aos metabólitos primários, estes correspondem à uma variedade de substâncias de distribuição ampla na natureza, sendo encontradas em quase todos os organismos, com a incumbência da manutenção e desenvolvimento da planta, podendo ser citados, as proteínas, açúcares, lipídeos e ácidos nucleicos. Para Santos (2007), os componentes do metabolismo secundário de uma planta, se referem a totalidade de substâncias que não participam essencialmente das necessidades do organismo produtor, porém, confere-a vantagens para a manutenção de sua sobrevivência, bem como de sua espécie.

Silva, 2008 e Dunder, 2009 exprimem que o resíduo líquido do sisal, abriga como principais componentes do seu metabolismo secundário, derivados alcaloides, cumarinas, taninos e saponínicos. Essa classe de metabólitos é detentora de funções terapêuticas, o que atrai o interesse farmacológico e comercial.

Para Puentes (2009) e Mazid et al., (2011), as saponinas participam do sistema de defesa do vegetal, protegendo-o do 
ataque de insetos, além de sua ação antifúngica e antibacteriana, principalmente contra microrganismos patogênicos. A classificação das saponinas se dá de duas maneiras: por meio do núcleo da aglicona, ou pelo seu caráter ácido, básico ou neutro. Referindo-se a aglicona, podem ser categorizadas em saponinas esteroidais ou triterpênicas.

No que se refere as saponinas esteroidais, Santos (2009) em consonância com López-Romero et al., (2018), subclassificam esta em: saponinas espirostanas, furostanas e esteroidal, os compostos dessa classe apresentam vastas propriedades farmacológicas.

Almeida (2013) retrata em seu estudo a constituição das saponinas esteroidais do sisal, afirmando que a sua classificação é variável, dependendo da fase de desenvolvimento em que a planta se encontra. Inicialmente, nas etapas que antecedem a biogênese das saponinas, tem-se a gitogenina, a qual é convertida em tigogenina, que no decorrer da vida da planta, resultará em hecogenina e neotigogenina, estas por sua vez, são as saponinas mais abundantes e comumente isoladas nas espécies de Agave. As saponinas esteroidais, são utilizadas na indústria farmacêutica como composto para a semi-síntese dos esteróides medicinais (esteroides diuréticos, hormônios sexuais e corticoides), apesar disso, alguns tipos de saponinas apresentam potencial de toxicidade aos seres humanos, como os glicoalcalóides esteroidais.

A investigação farmacológica do suco das folhas de Agave sisalana ratificou a sua ação no tratamento de sífilis, doenças da pele e como laxante. A seiva desta planta valida propriedades antissépticas, sendo útil na contenção de processos infecciosos do trato digestivo. Ainda assim, atua no estímulo da musculatura uterina e intestinal, culminando na diminuição da pressão sanguínea e consequente aborto (animais gestantes) (Debnath et al., 2010). A Tabela 2 descreve as principais atividades biológicas do sisal.

Tabela 2: Atividades biológicas de Agave sisalana.

\begin{tabular}{|c|c|c|c|}
\hline Autor/Ano & Título & Atividade & Descrição \\
\hline $\begin{array}{l}\text { Keriko \& Mutua } \\
\qquad(\mathbf{2 0 0 8 )}\end{array}$ & $\begin{array}{l}\text { Inseticidal activities of the } \\
\text { sisal plant, Agave sisalana, } \\
\text { Agavaceae extracts against } \\
\text { white termites, } \\
\text { Reticulitermes flavipes } \\
\text { (Kollar) Rhinotermitidae }\end{array}$ & Inseticida & $\begin{array}{c}\text { A ação inseticida do extrato das } \\
\text { folhas do sisal foi testada em } \\
\text { Reticulitermes flavipes (cupim } \\
\text { subterrâneo), onde a concetração de } \\
1000 \text { ppm dos extratos metanólico, } \\
\text { diclorometano e n-hexanol } \\
\text { mostraram eficácia. }\end{array}$ \\
\hline $\begin{array}{c}\text { Raju \& Mehta } \\
\text { (2009) }\end{array}$ & $\begin{array}{l}\text { Cancer chemopreventive } \\
\text { and therapeutic effects of } \\
\text { diosgenin, a food saponin }\end{array}$ & $\begin{array}{l}\text { Quimioprevenção e } \\
\text { controle do câncer }\end{array}$ & $\begin{array}{l}\text { Neste estudo, a atividade da } \\
\text { diosgenina (um tipo de saponina) } \\
\text { demonstrou função antineoplásica, } \\
\text { por meio da modulação de vários } \\
\text { eventos de sinalização celular } \\
\text { (crescimento, diferenciação, } \\
\text { apoptose e oncogênese). }\end{array}$ \\
\hline $\begin{array}{l}\text { Silveira et al., } \\
\qquad(2009)\end{array}$ & $\begin{array}{l}\text { Influência do resíduo } \\
\text { líquido do sisal (Agave } \\
\text { sisalana, Perrine) sobre a } \\
\text { alimentação larvar e } \\
\text { motilidade de adultos, in } \\
\text { vitro, de nematóides } \\
\text { gastrintestinais de pequenos } \\
\text { ruminantes. }\end{array}$ & Antiparasitária & $\begin{array}{l}\text { Estudo que comprova a ação } \\
\text { antiparasitária de Agave sisalana por } \\
\text { meio da utilização do resíduo líquido } \\
\text { de suas folhas, mediante testes, foi } \\
\text { possível inibir em } 100 \% \text { a } \\
\text { alimentação larval, bem como a } \\
\text { motilidade de nematóides } \\
\text { gastrintestinais de ovinos e caprinos. }\end{array}$ \\
\hline $\begin{array}{l}\text { Dunder et al., } \\
\qquad(2010)\end{array}$ & $\begin{array}{c}\text { Potencial antiinflamatório e } \\
\text { analgésico do extrato } \\
\text { hidrolisado de Agave } \\
\text { sisalana Perrine ex } \\
\text { Engelm., Asparagaceae }\end{array}$ & $\begin{array}{c}\text { Anti-inflamatória e } \\
\text { analgésica }\end{array}$ & $\begin{array}{l}\text { Testagem das propriedades anti- } \\
\text { inflamatória e analgésicas do extrato } \\
\text { hidrolisado de Agave sisalana em } \\
\text { modelos clássicos de inflamação, } \\
\text { demosntrando como resultados, a } \\
\text { diminuição da inflamação no tecido } \\
\text { granulomatoso, expressando as } \\
\text { atividades anti-inflamatória e }\end{array}$ \\
\hline
\end{tabular}




\begin{tabular}{|c|c|c|c|}
\hline & & & $\begin{array}{l}\text { analgésica desta planta em processos } \\
\text { agudos e crônicos. }\end{array}$ \\
\hline $\begin{array}{l}\text { Abreu } \\
(2013)\end{array}$ & $\begin{array}{c}\text { Efeitos comportamentais, } \\
\text { neuroquímicos e } \\
\text { antioxidantes da hecogenina } \\
\text { obtida de Agave sisalana } \\
\text { Perrine em camondongos }\end{array}$ & $\begin{array}{c}\text { Efeitos } \\
\text { comportamentais e } \\
\text { neuroquímicos }\end{array}$ & $\begin{array}{l}\text { Estudo in vitro realizado em } \\
\text { camundongos, os quais foram } \\
\text { submetidos a atividade das } \\
\text { hecogeninas de } A \text {. sisalana. Os } \\
\text { resultados demostraram o } \\
\text { comprovado efeito ansiolítico da } \\
\text { planta, sem que houvesse estresse } \\
\text { oxidativo no sistema nervoso central. }\end{array}$ \\
\hline $\begin{array}{l}\text { Botura et al., } \\
\qquad(2013)\end{array}$ & $\begin{array}{l}\text { In vitro ovicidal and } \\
\text { larvicidal activity of Agave } \\
\text { sisalana Perr. (sisal) on } \\
\text { gastrointestinal nematodes } \\
\text { of goats }\end{array}$ & Ovicida & $\begin{array}{l}\text { Avaliação da atividade ovicida in } \\
\text { vitro de Agave sisalana, observada } \\
\text { em frações de flavonóides, extratos } \\
\text { aquosos e acetato de etila. }\end{array}$ \\
\hline $\begin{array}{l}\text { Zhang et al., } \\
\qquad(2013)\end{array}$ & $\begin{array}{c}\text { Structural features, } \\
\text { antioxidant and } \\
\text { immunological activity of a } \\
\text { new polysaccharide (SP1) } \\
\text { from sisal residue }\end{array}$ & Imunomodulador & $\begin{array}{l}\text { Indicação do resíduo do } \\
\text { desfibramento das folhas de } A \text {. } \\
\text { sisalana, como importante agente } \\
\text { para a indústria farmacêutica, } \\
\text { destacando sua atividade } \\
\text { imunomoduladora. }\end{array}$ \\
\hline $\begin{array}{l}\text { Vieira et al., } \\
\text { (2014) }\end{array}$ & $\begin{array}{l}\text { Avaliação das atividades } \\
\text { antimicrobiana, } \\
\text { antioxidante e de } \\
\text { citotoxidades de produtos } \\
\text { extraídos da Agave sisalana } \\
\text { Perrine }\end{array}$ & Antifúngica & $\begin{array}{l}\text { Neste estudo foi avaliada a } \\
\text { efetividade dos extratos etanólicos } \\
\text { de Agave sisalana sob cepas de } \\
\text { Candida albicans, o qual mostrou-se } \\
\text { eficiente na inibição deste fungo, em } \\
\text { concentrações inibitórias } \\
\text { recomendadas para plantas } \\
\text { medicinais. }\end{array}$ \\
\hline $\begin{array}{l}\text { Nunes et al., } \\
\qquad \text { (2015) }\end{array}$ & $\begin{array}{l}\text { The larvicidal activity of } \\
\text { Agave sisalana against L4 } \\
\text { larvae of Aedes aegypti is } \\
\text { mediated by internal } \\
\text { necrosis and inhibition of } \\
\text { nitric oxide production }\end{array}$ & Larvicida & $\begin{array}{c}\text { O extrato bruto de } A \text {. sisalana foi } \\
\text { descrito como agente larvicida de } \\
\text { eficácia contra larvas de Aedes } \\
\text { aegypti, expressando em ensaios } \\
\text { atividade necrotizante em hemócitos, } \\
\text { com inibição da produção de óxido } \\
\text { nítrico. }\end{array}$ \\
\hline $\begin{array}{l}\text { López-Romero et al., } \\
\qquad(2018)\end{array}$ & $\begin{array}{l}\text { Biological activities of } \\
\text { Agave by-products and their } \\
\text { possible applications in } \\
\text { food and pharmaceuticals }\end{array}$ & Antibacteriana & $\begin{array}{l}\text { A ação antibacteriana da Agave } \\
\text { sisalana, é mediada por mudanças } \\
\text { nas propriedades da membrana } \\
\text { celular da bactéria (gram-positivas } \\
\text { ou negativas), provocando } \\
\text { modificações em sua integridade, o } \\
\text { que resulta no vazamento do } \\
\text { material intracelular e morte. }\end{array}$ \\
\hline $\begin{array}{l}\text { De Oliveira et al., } \\
\qquad(2019)\end{array}$ & $\begin{array}{l}\text { Saponin-rich fraction from } \\
\text { Agave sisalana: effect } \\
\text { against malignant astrocytic } \\
\text { cells and its chemical } \\
\text { characterisation by ESI- } \\
\text { MS/MS }\end{array}$ & Anticâncer & $\begin{array}{l}\text { Células tumorais astrocíticas } \\
\text { derivadas de gliomas de ratos e de } \\
\text { humanos, além de células } \\
\text { astrocíticas não tumorais foram } \\
\text { expostas à fração rica em saponina } \\
\text { do resíduo de Agave sisalana o que } \\
\text { elucidou seu efeito citotóxico, de } \\
\text { modo que houve redução da } \\
\text { viabilidade celular com uma maior } \\
\text { intensidade ao glioma. }\end{array}$ \\
\hline $\begin{array}{l}\text { Barreto et al., } \\
\qquad(2020)\end{array}$ & $\begin{array}{l}\text { In Vitro and In Vivo } \\
\text { Antioxidant Activity of } \\
\text { Agave sisalana Agro- } \\
\text { Industrial Residue }\end{array}$ & Antioxidante & $\begin{array}{l}\text { O resíduo agroindustrial da Agave } \\
\text { sisalana foi testado in vitro e in vivo, } \\
\text { demostrando assim, potencial contra } \\
\text { danos associados ao estresse } \\
\text { oxidativo e ao envelhecimento. }\end{array}$ \\
\hline
\end{tabular}


Na Tabela 2 estão contidos informes sobre a variedade de ações biológicas mediadas pela Agave sisalana. Keriko \& Mutua (2008), apontam sua atividade inseticida, Raju e Mehta (2009), seu potencial na quimioprevenção e controle do câncer, Silveira et al., (2009), seu uso como antiparasitária, Dunder et al., (2010), como anti-inflamatório e analgésico. Abreu (2013), por sua vez, descreve os seus efeitos sob a atividade comportamental e neuroquímica, Botura et al., (2013), como ovicida, Zhang et al., (2013), imunomodulador, Vieira et al., (2014), antifúngico, Nunes et al., (2015), larvicida, López-Romero et al., (2018), antibacteriano, De Oliveira et al., (2019), anticâncer e Barreto et al., (2020) como antioxidante.

Além das atividades biológicas voltadas para as competências farmacológicas, Barrêto et al., (2010) retratam a utilização da A. sisalana na agricultura, onde após maceração e 24 horas de incubação, o extrato da planta foi aplicado no ácaro rajado e Iphiseiodes spp., para avaliação dos seus efeitos, observando-se então, uma diminuição significativa do número de ácaros vivos. Em consonância, o estudo realizado por Costa (2012), demonstrou a eficácia do extrato do sisal no controle da praga Spodoptera frugiperda. Souza (2009), por sua vez, cita a efetividade deste, sob o mosquito Culex quinquefasciatus, mostrando a sua eficácia como inseticida.

O estudo realizado por Cavalcante et al., (2017) investigou o uso da A. sisalana como fertilizante, o resíduo da mesma foi empregado em gravioleiras, por meio da irrigação e da cobertura morta do solo, para que houvesse posteriormente, uma verificação da qualidade dos frutos. Os resultados mostraram que a utilização na forma de irrigação foi a mais aceitável, culminando em uma maior produção, com aumento da massa dos frutos. As concentrações finais de sacarose, glicose e vitamina $\mathrm{C}$ das polpas foram investigadas, demonstrando satisfatoriedade em seus percentuais. Para Martin et al., (2009) o uso da Agave sisalana na agropecuária se dá também, por meio da sua aplicação na ração de animais.

\subsection{Perfil antimicrobiano de Agave sisalana}

A mensuração de determinados compostos em plantas, com atividade antimicrobiana tem sido objeto de atratividade para a comunidade científica. Substâncias como citral, cineol, flavonoides, limoneno e terpenos, são apontados como possuintes de efeito antagônico sob microrganismos (Almeida, 2013).

Para Santos et al., (2015) a atividade antimicrobiana da Agave sisalana vincula-se a sua composição, sendo atribuída aos compostos fenólicos, terpenos e saponinas, com ênfase para as saponinas esteroidais as quais apresentam maior concentração, vinculando-se assim, aos seus efeitos biológicos.

Em um estudo realizado por Hammuel et al., (2011), foram avaliadas as propriedades fitoquímicas, bem como a atividade antimicrobiana da Agave sisalana. Na análise fitoquímica foi elucidada a presença de metabólitos secundários, como: esteroides, saponinas, glicosídeos, taninos e flavonóides. No que se referiu a ação antimicrobiana in vitro, o extrato aquoso demonstrou capacidade antimicrobiana, inibindo o crescimento de microrganismos.

Vieira (2014), em sua investigação, elucidou a atividade antibacteriana e antifúngica da Agave sisalana, ao utilizar os resíduos de decorticação da planta. A atividade antibacteriana foi determinada em cepas de Escherichia coli, Pseudomonas aeruginosa e Staphylococcus aureus. No que se destina a ação antifúngica, esta foi comprovada contra a Candida albicans, caracterizando um novo ativo terapêutico.

Em relação a atividade antifúngica, Costa (2012) relatou em seu estudo, que as folhas da Agave sisalana, apresentaram funcionalidade inibitória contra os fungos: Saccharomyces cerevisiae, Crebrothecium ashbyi e Cryptococcus neoformans, entretanto, uma atividade diminuída foi apontada para os fungos: Aspergillus oryzae, Aspergillus flavus e Penicillium chrysogenum.

Almeida (2013) explana a eficácia antimicrobiana comprovada do extrato de A. sisalana, onde em sua investigação, uma concentração inibitória mínima (CIM) de $98 \mu \mathrm{g} / \mathrm{mL}$ foi detectada para Candida albicans, $783 \mu \mathrm{g} / \mathrm{mL}$ para o Tricophyton mentagrophytes e de $3125 \mu \mathrm{g} / \mathrm{mL}$ para Malassezia pachydermatis. Morais e colaboradores (2010), concluíram que o extrato da 
A. sisalana demonstrou inibição sob Fusarium oxysporum.

Santos et al., (2009) no que lhes concernem, testificaram a atividade antifúngica da Agave sisalana a partir do uso do extrato das folhas e resíduos obtidos pelo processo de aquisição das fibras duras, afirmando o potencial do extrato hidroetanólico na inibição de Candida albicans.

Verástegui et al., (2008) atestam os efeitos de várias espécies de Agave contra bactérias patogênicas e fungos. A Agave picta foi aplicada em colônias de Staphylococcus aureus, Escherichia coli, Listeria monocytogenes e Vibrio cholerae sendo explicita a inibição, porém, os efeitos desta nos Bacillus cereus e Yersinia enterocolitica, não apresentou eficiência. Outro aspecto de importância, é que todas as espécies de Agave do estudo (A. lecheguilla, A. picta, A. scabra e A. lophanta), foram inibitórias para a variedade de fungos e leveduras analisadas, demonstrando assim, que a atividade terapêutica não se restringe apenas para a espécie sisalana, estendendo-se para outras, pertencentes ao gênero Agave.

\section{Conclusão}

Com base nos materiais utilizados para a construção deste estudo, foi possível elucidar a variedade de aplicações que a Agave sisalana possibilita, seja de cunho industrial, artesanal na agropecuária ou no âmbito terapêutico. A sua atividade antimicrobiana na inferência de fungos ou bactérias é cientificamente comprovada, fazendo desta uma importante alternativa, frente a incidência de microrganismos resistentes as formulações disponíveis até então.

Cabe frisar, a relevância desta planta para a economia das regiões que a cultivam, destacando a região sisaleira da Bahia, como a cidade de Retirolândia, a qual exporta uma variedade de materiais advindos do sisal. Mais estudos devem ser destinados ao aproveitamento completo de seu resíduo líquido, a fim usufruir de todos os benefícios farmacológicos desta, além de alavancar a sua comercialização pela indústria farmacêutica, uma vez que se trata de uma matéria prima barata com diversidade em uso.

\section{Referências}

Abreu, R. N. D. C. (2013). Efeitos comportamentais, neuroquímicos e antioxidante da hecogenina obtida de Agave sisalana Perrine em Camundongos. $164 \mathrm{f}$. Tese (Doutorado em Biotecnologia) - Centro de Ciências, Universidade Federal do Ceará, Fortaleza.

Almeida, E. C. D. S. L. (2013). Ação antifúngica do resíduo líquido integral e fracionado do desfibramento da folha do sisal (Agave sisalana) sobre Malassezia pachydermatis, Tricophyton mentagrophytes e Candida albicans. 94 f. Dissertação (Mestrado em Ciências Biológicas - Microbiologia Aplicada) Instituto de Biociência de Rio Claro, Universidade Estadual Paulista, Rio Claro.

Barrêto, A. F., Araújo, E., \& Bonifácio, B. F. (2010). Eficiência de extratos de Agave sisalana (Perrine) sobre o ácaro rajado Tetranychus urticae (Koch) e ocorrência de fitotoxidez em plantas de algodoeiro (Gossypium hirsutum L. r latifolium Hutch). Revista Brasileira de Agroecologia, 5(2), 207-215.

Barreto, S. M. A. G. (2017). Utilização do subproduto do beneficiamento do sisal (Agave sisalana Perrine): desenvolvimento de nanoemulsões cosméticas e avaliação da segurança e eficácia. 117f. Dissertação (Mestrado em Ciências Farmacêuticas) - Centro de Ciências da Saúde, Universidade Federal do Rio Grande do Norte, Natal.

Barreto, S. M. A. G., Cadavid, C. O. M., Moura, R. A. D. O., Silva, G. M. M., Araújo, S. V. F. D., Silva Filho, J. A. A. D., \& Ferrari, M. (2020). In vitro and in vivo antioxidant activity of Agave sisalana agro-industrial residue. Biomolecules, 10(10), 1435.

Botura, M. B., dos Santos, J. D. G., da Silva, G. D., de Lima, H. G., de Oliveira, J. V. A., de Almeida, M. A. O., \& Branco, A. (2013). In vitro ovicidal and larvicidal activity of Agave sisalana Perr.(sisal) on gastrointestinal nematodes of goats. Veterinary Parasitology, 192(1-3), 211-217.

Branco, A. et al. (2010). D-mannitol from Agave sisalana biomass waste. Industrial Crops and Products, 32(1), 507-510.

Cardoso, M. S. (2019). Panorama tecnológico de uso de resíduos do sisal. Semioses, 13(3), 13-23.

Carneiro, J. L. D. S. (2016). Caracterização acessos de sisal usando descritores da planta e da fibra. 118 f. Tese (Doutorado Acadêmico em Recursos Genéticos Vegetais) - Universidade Estadual de Feira de Santana, Feira de Santana.

Cavalcante, L. F., da Rocha, L. F., Silva, R. A. R., Souto, A. G. L., Nunes, J. C., \& Cavalcante, Í. H. L. (2017). Produção e qualidade da graviola sob irrigação e cobertura do solo com resíduo de sisal. Magistra, 28(1), 91-101.

Costa, M. F. (2012). Aproveitamento do resíduo líquido industrial do sisal (Agave sisalana Perr.) para obtenção de um inseticida biológico. 92 f. Dissertação (Mestrado em Recursos Genéticos Vegetais) - Universidade Estadual de Feira de Santana, Feira de Santana 
De Oliveira, J., Botura, M. B., Dos Santos, J., Argolo, D. S., da Silva, V., da Silva, G. D., de Lima, H. G., Braz Filho, R., Vieira, I., Branco, A., Batatinha, M., \& Costa, S. L. (2019). Saponin-rich fraction from Agave sisalana: effect against malignant astrocytic cells and its chemical characterisation by ESI-MS/MS. Natural product research, 33(12), 1769-1772.

Debnath, M., Mukeshwar, P., Sharma, R., Thakur, G. S., \& Lal, P. (2010). Biotechnological intervention of Agave sisalana: a unique fiber yielding plant with medicinal property. Journal of Medicinal Plants Research, 4(3), 177-187.

Dunder, Ricardo J., Quaglio, Ana EV, Maciel, Rubens P., Luiz-Ferreira, Anderson, Almeida, Ana CA, Takayama, Christiane, Faria, Felipe M. de, \& SouzaBrito, Alba RM. (2010). Potencial antiinflamatório e analgésico do extrato hidrolisado de Agave sisalana Perrine ex Engelm. Asparagaceae. Revista Brasileira de Farmacognosia, 20(3), 376-381.

Hammuel, C., Yebpella, G. G., Shallangwa, G. A., Magomya, A. M., \& Agbajp, A. S. (2011). Phytochemical and antimicrobial screening of methanol and aqueous extracts of Agave sisalana. Acta poloniae pharmaceutica, 68(4), 535-539.

Keriko, J. M., Mutua, M. M. (2008). Inseticidal activities of the sisal plant, Agave sisalana, Agavaceae extracts against white termites, Reticulitermes flavipes (Kollar) Rhinotermitidae. Journal of Agriculture, Science and Technology, 10(1), 70-75.

Leal, T. T. B. (2016). Bioatividade do resíduo de sisal (Agave sisalana Perrine ex Engelm) sobre Ceratitis capitata (Wiedemann, 1824) (Diptera: Tephritidae) e Diachasmimorpha longicaudata (Ashmead, 1905) (Hymenoptera: Braconidae). Dissertação (Mestrado em Ciências Agrárias - Fitotecnia). Universidade Federal do Recôncavo da Bahia, Cruz das Almas.

Lin, K. W. et al. (2015). Antioxidant prenylated phenols of Artocarpus plants attenuate ultraviolet radiation-induced damage on human keratinocytes and fibroblastos. Phytochemistry Letters, 14(1), 190-197.

López-Romero J. C., AYALA-Zavala J. F., González-Aguilar G. A., Peña-Ramos E. A., González-Ríos H. (2018). Biological activities of Agave by-products and their possible applications in food and pharmaceuticals. J Sci Food Agric, 98(7)2461-2474.

Maran, J. P, Priya, B. (2015). Ultrasoubd-assisted extraction of pectin from sisal waste. Carbohydrate Polymers, 115(1),732-738.

Martin, A. R., Mattoso, M. A. M., Mattoso, L. H. C., Silva, O. R. R. F. Silva (2009) Caracterização química e estrutural de fibra de sisal da variedade Agave sisalana. Polímeros: Ciência e Tecnologia, 19(1), 40-46.

Mazid, M., Khan, T. A., \& Mohammad, F. (2011). Role of secondary metabolites in defense mechanisms of plants. Biology and medicine, 3(2), $232-249$.

Morais, M. S., Araújo, E., Araújo, A. C., Belém, L. F. (2010). Eficiência dos extratos de alho e agave no controle de Fusarium oxysporum S. Rev. Bras. de Agroecologia. 5(2), 89-98.

Nunes, F. C., Leite, J. A., Oliveira, L. H., Sousa, P. A., Menezes, M. C., Moraes, J. P., Mascarenhas, S. R., \& Braga, V. A. (2015). The larvicidal activity of Agave sisalana against L4 larvae of Aedes aegypti is mediated by internal necrosis and inhibition of nitric oxide production. Parasitology research, 114(2), 543-549.

Pereira, A. S., Shitsuka, D. M., Parreira, F. J., \& Shitsuka, R. (2018). Metodologia da pesquisa científica. UFSM. https://repositorio.ufsm.br/bitstream /handle/1/15824/Lic_Computacao_MetodologiaPesquisa-Cientifica. pdf.

Puentes, L. N. D. (2009). Interacciones moleculares entre plantas y microorganismos: saponinas como defensas químicas de las plantas y su tolerancia a los microorganismos. Una revisión. RET. Revista de Estudios Transdisciplinarios, 1(2), 32-55.

Raju, A., \& Mehta, R. (2009). Cancer chemopreventive and therapeutic effects of diosgenin, a food saponin. Nutrition and cancer. 61(1), 27-35.

Santos, J. D. G. (2009). Avaliação da atividade antimicrobiana de extratos e caracterização parcial de saponinas obtidas do resíduo de Agave sisalana Perrine (Sisal). 113 f. Dissertação (Mestrado em Recursos Genéticos Vegetais) - Universidade Estadual de Feira de Santana, Feira de Santana.

Santos, R. I., Simões, C. M. O., Schenkel, E. P., Gosmann, G., Mello, J. C. P. (2007). Metabolismo básico e origem dos metabólitos secundários, Farmacognosia: da planta ao medicamento. Florianópolis: Editora da UFSC. 1104 p.

Santos, J. D., Vieira I. J., BRAZ-FILHO R., Branco A. (2015). Chemicals from Agave sisalana biomass: isolation and identification. Int J Mol Sci. 16(4),876171 .

Silveira, R. X., Chagas, A. D. S., Botura, M. B., Batatatinha, M., Katiki, L., Bevilaqua, C., \& Ornelas-Almeida, M. A. (2009). Influência do resíduo líquido do sisal (Agave sisalana, Perrine) sobre a alimentação larvar e motilidade de adultos, in vitro, de nematóides gastrintestinais de pequenos ruminantes. In Embrapa Pecuária Sudeste - Artigo em anais de congresso. In: Congresso Brasileiro de medicina veterinária, conbravet.

Souza-Júnior, E. F. (2017). Modelagem e Simulação fenomenológica de biorreator para a produção de xilitol a partir de sisal (Agave sisalana) em meio com Candida guilliermondii. João Pessoa. Monografia (Curso de Graduação em Engenharia Química) Campus I - UFPB / Universidade Federal da Paraíba.

Souza M. F. (2009). Atividade inseticida de extratos obtidos a partir do resíduo líquido de agave sisalana perrine no controle da praga Spodoptera frugiperda (J.E. Smith, 1797) (Lepidoptera: noctuidae) em milho. 77 f. Dissertação (Mestrado em Biotecnologia) - Universidade Estadual de Feira de Santana, Feira de Santana.

Taiz, L., \& Zeiger, E. (2013). Fisiologia vegetal. (5a ed.), Artmed. 918p.

Verástegui, A., Verde, J., García, S., Heredia, N., Oranday, A. \& Rivas, C. (2008). Species of Agave with antimicrobial activity against selected patogenic bactéria and fungi. World J. Microbiol. Biotechnol. 24(1), 1249-1252.

Vieira, J. P. L. (2014). Avaliação das atividades antimicrobiana, antioxidante e de citotoxidades de produtos extraídos da Agave sisalana Perrine. 65f. Dissertação (Programa de Pós-Graduação em Ciências Farmacêuticas - PPGCF) - Universidade Estadual da Paraíba, Campina Grande. 
Research, Society and Development, v. 10, n. 3, e2510312734, 2021

(CC BY 4.0) | ISSN 2525-3409 | DOI: http://dx.doi.org/10.33448/rsd-v10i3.12734

Vuorinne, I., Heiskanen, J., \& Pellikka, PK (2021). Avaliando a biomassa foliar de Agave sisalana usando índices de vegetação Sentinel-2. Sensoriamento remoto, 13 (2), 233.

Zhang, X., Liu, L., Lin C. (2013). Structural features, antioxidant and immunological activity of a new polysaccharide (SP1) from sisal residue. International Journal of Biological Macromolecules. 59(1), 184-191. 